\title{
Der Basler Arzt Theodor Zwinger III (1658-1724) und seine Arbeit über ein langes Leben
}

Von Marie-Louise Portmann

Im Jahre 1966 veröffentlichte der Historiker Gerald J.Gruman von der University of Massachusetts eine ideengeschichtliche Studie, betitelt "A History of ideas about the prolongation of life". Diese Arbeit erschien in den "Transactions of the American Philosophical Society held at Philadelphia" ${ }^{1}$. Der Autor geht das Thema der Langlebigkeit vom philosophischen Standpunkt an und bezieht auch fernöstliches Gedankengut ein. In Kapitel VII spricht er von den Hygienikern, an deren Spitze er den venezianischen Patrizier Lodovico Cornaro stellt, der von 1467 bis 1566 lebte. Dieser gab in hohem Alter eine Darstellung maßvoller Lebensweise heraus, betitelt «Discorsi della vita sobria». Gruman zählt zu den Hygienikern auch Christoph Wilhelm Hufeland, der im Jahre 1797 sein Buch über «Die Kunst das menschliche Leben zu verlängern» veröffentlichte. Zwischen Cornaro und Hufeland ist der Basler Mediziner Theodor Zwinger anzusiedeln, der sich 1703 für das Amt eines Professors für Anatomie und Botanik bewarb und zu diesem Zweck seine Abhandlung «De adquirenda vitae longaevitate» ausarbeitete $^{2}$.

Sehen wir uns die Ratschläge Zwingers näher an, ein hohes Alter zu erreichen. Dabei spielt bei ihm, wie wir sehen werden, die Diät eine große Rolle. Es ist zu beachten, daß Zwinger unter Diät noch ganz im Sinne des hippokratischen Begriffs «diaita» die ganze Lebensweise versteht, also nicht nur Nahrung, sondern auch Beschäftigung, Wohnung und Aufenthalt. Ja, die Sorge für ein hohes Alter der Kinder soll von den Eltern bereits bei der Eheschließung beginnen. Bei der Zeugung sollen die Eltern ihre Konstitution prüfen ${ }^{3}$, ob sie nüchtern, sorglos, leidenschaftslos und heiteren Geistes und auch nicht übermüdet seien. Auch dürfen sie nicht krank sein, damit die Kinder nicht erblich belastet sind. Denn so wie die Eltern sind, werden auch ihre Kinder werden. Zwinger rät allen Verlobten an, vor der Vermählung ihre Körper zu purgieren, um alle Schlacken auszutreiben ${ }^{4}$. Auch soll eine gute Diät eingehalten und die Seele heiter gestimmt werden.

Sobald die Mutter empfangen hat, soll sie sich besonders vor schlechter Luft, schweren, scharfen oder zu salzigen Speisen hüten. Sie darf sich auch nicht durch Genuß von unreifem Obst den Magen verderben, sonst sei ein 
Abort zu befürchten. Heftige Bewegungen und auch psychische Aufregungen seien zu vermeiden. Zwinger schreibt auch alle 2-3 Monate den Aderlaß vor und empfiehlt verschiedene Medikamente, die den Foetus stärken sollen ${ }^{5}$. Der Gatte soll sich um die Schwangere kümmern und durch freundlichen Umgang mit ihr günstige Bedingungen schaffen.

Wenn das Kind geboren ist, soll es zuerst äußerlich gut gereinigt werden, sodann soll dem Säugling zwecks Abgangs des Mekoniums für 24 Stunden oder auch einige Tage ungesalzene frische Butter, ganz frisches Mandelöl mit ein wenig Veilchensyrup, Honig oder Korallenpulver verabreicht werden ${ }^{6}$. Die Mutter möge dann nach Einnahme zweckmäßiger Stärkungsmittel den Säugling sogleich stillen, wobei Zwinger betont, daß das Colostrum nicht schädlich sei, sondern mit dazu diene, das Mekonium auszutreiben. Zwinger warnt davor, dem Säugling Brühen zu verabreichen. In den ersten Wochen und Monaten soll er nichts anderes als Muttermilch bekommen, später sollen ihm dann allmählich Speisen verabreicht werden, eine Suppe aus Weizenmehl und Kuhmilch, die aber nicht fett sein darf. Anfangs soll der Speisezusatz zur Muttermilch einmal täglich, sodann zwei- bis dreimal täglich verabreicht werden, bis der Säugling entwöhnt ist. Wir sehen, um ein hohes Alter zu erreichen, bemüht sich Zwinger, der Kinderarzt, zunächst die Säuglingssterblichkeit herabzusetzen.

Zwinger gibt dann auch verschiedene Präventivmittel und Rezepte gegen Konvulsionen der Kinder an. Auch müsse man sehr darauf achten, $\mathrm{da} ß$ die Muttermilch von guter Beschaffenheit sei. Sie darf nicht scharf oder fett sein. Der Basler Arzt kennt ein kompliziertes Rezept zur Verbesserung der Muttermilch ${ }^{7}$. Wenn dies nicht hilft, muß eine Amme beigezogen werden, wobei aber wieder darauf geachtet werden muß, daß es eine gesittete und anständige Person sei. Wenn eine Amme nicht vorhanden sei, müsse man sich mit Brühen und Wasser behelfen, doch sei dann für das Kind kein langes Leben zu erhoffen ${ }^{8}$. Alle 8-14 Tage soll das Kind in Wasser gebadet werden, dem aber Wein und Milch und verschiedene Heilkräuter beizumischen sind. Nach der Entwöhnung sollen die Kinder Milchsuppen bekommen, und dann verabreiche man ganz allmählich auch feste Speisen, wobei aber alles Scharfe vermieden werden muß, um Hautkrankheiten vorzubeugen. Kinder sollen keine Schleckereien bekommen, sondern an einfache Nahrung gewöhnt werden. Auch sollen sie nicht müßig sein, sondern zur Arbeit angehalten werden. Aller Luxus soll von ihnen ferngehalten werden, so daß sie zur Sparsamkeit erzogen werden. Begabte Kinder sollen zum Lernen angehalten werden, jedoch ohne daß man einen Druck auf sie ausübt. 
Allmählich sollen das Gedächtnis und der Geist trainiert werden, aber ohne Zwang, denn wie Zwinger sagt, die Natur lasse sich nicht zwingen, sondern wolle geführt werden ${ }^{9}$. Fleißige Kinder sollen belohnt werden, um ihren Ehrgeiz anzustacheln, die Nachlässigen sollen mit Maß und Gerechtigkeit gezüchtigt werden. Die Nahrung sei abwechselnd und sorgfältig zubereitet. Als Getränk für Kinder diene Wasser oder ungegorenes Bier. Bisweilen könne man ein kleinwenig Wein erlauben, aber Schnaps sei verboten. Der Schlaf soll acht oder mehr Stunden dauern, daneben sollen auch mit Maß körperliche Übungen betrieben werden. Soweit äußert sich Zwinger, der sich als Pädiater auszeichnete, über die Vorkehrungen zur Langlebigkeit im Kindesalter.

Aber er befaßt sich in seiner Schrift auch mit der Lebenshaltung der Erwachsenen und der Greise. Besonders viel verspricht sich $Z$ winger von einem zeitweiligen Luftwechsel, da er der reinen und leichten Luft besondere Wirkung für die Kräftigung der Lungen und für die Regelung der Verdauung zuschreibt ${ }^{10}$. Er preist vor allem das Klima der Schweiz und empfiehlt vornehmlich jene Orte, wo Heilquellen entspringen. Zwinger geht dann dazu über, die gesunde Lage Basels zu preisen, wo viele kristallklare Quellen entspringen. Auch gebe es viele Plätze zum Lustwandeln, so den Petersplatz, die Rheinbrücke und die Pfalz. Auch seien viele schöne Spazierwege vor den Toren der Stadt, von wo man prächtige Ausblicke auf die Umgegend genieße. Nahe der Stadt gebe es Wiesen, Hügel und Wälder, wo man sich erholen könne und Vogelgesang höre. Wer Flüsse bevorzuge, finde in Basel nicht nur den Rhein, der die Stadt in zwei Teile teile, sondern auch die Birs, den Birsig und die Wiese. Daneben gebe es in der Stadt viele abgeleitete Flußläufe, welche Mühlen treiben, und in den Gewässern seien die besten Fische zu fangen. Darum müsse man sich nicht wundern, daß viele Fremde nach Basel kommen, um sich hier eine Zeitlang aufzuhalten und sich zu erholen ${ }^{11}$.

Auch in seinem Haus soll jeder, je nach der Jahreszeit, verschiedene Gemächer bevorzugen, um das beste Klima zu genießen. Im Winter und Frühling soll man in Zimmern wohnen, die nach Osten und Süden gerichtet sind, während man im Sommer und Herbst gegen Norden und Westen wohnen möge. Zwinger lobt dann die Sitte der Fürsten, sich nicht nur in ihren Palästen verschiedene Zimmer einzurichten, um sie je nach der Jahreszeit zu wechseln, sondern sich an verschiedenen Orten anders gelegene Gebäude zu errichten, um je nach Bedarf den Wohnsitz zu wechseln. Jedoch könne der gewöhnliche Sterbliche nicht immer die beste Luft genießen. 
Zwinger erwähnt den üblen Geruch von Misthaufen und schlecht gereinigten Kloaken, auch von Kadavern, die zu wenig tief vergraben worden seien. Auch die üble Luft über stehenden Gewässern und Sümpfen sei schädlich ${ }^{12}$. Der Autor gibt dann Ratschläge, wie man sich vor feuchter Luft schützen könne. Das Entzünden von Eichen-, Tannen- oder Buchenholz schaffe gute Abhilfe. Auch das Tabakrauchen empfiehlt er. Schließlich könne man den Kachelofen heizen. So schütze man sich vor Pestluft, denn die Flammen verbrennen die giftigen Partikelchen. Auch das Räuchern mittels Weihrauch, Mastix und andern wohlduftenden Ingredienzien sei ein gutes Mittel gegen ansteckende und pestbringende Luft. Darüber hinaus rät Zwinger, die Steinfliesen der Häuser prophylaktisch mit scharfen Substanzen zu besprühen, wobei es sich hauptsächlich um pflanzliche Dekokte handelte, deren Zusammensetzung er genau angibt. Trockene und warme Luft, welche die Körpersäfte zersetze und scharf mache, könne man korrigieren, indem man die Böden oft mit kaltem Wasser übergieße. Auch die Blätter gewisser Pflanzen (Reben-, Eichenblätter usw.) könne man aufstreuen, wobei man aber die Hitze so gut als möglich abschirmen solle. Kalte Luft, welche die Körpersäfte eindickt, wird durch Feuer gemildert, sei es, daß man es in den Kaminen entzünde, sei es, daß man den Ofen heize. Doch müsse man sich hüten, kalte Gliedmaßen sofort am Feuer zu erwärmen, denn die Hitze bewirke eine Zusammenziehung der Körperfasern, was die Säftezirkulation störe und zu Gangrän führe. Schlecht riechende Luft soll durch Räucherung mit wohlriechenden Stoffen gebessert werden, welche man in den Apotheken in Form von Flüssigkeiten, Täfelchen, Kerzen oder Harzen bekommen könne ${ }^{13}$.

Was die Winde anbelange, so seien der West- und Nordwind besonders schädlich, weil sie die Zirkulation der Lebenssäfte stören, darum soll man sich nicht lange diesen Winden aussetzen. Besonders die Greise müssen sich davor schützen und morgens nicht ausgehen, bevor die Sonnenstrahlen die Nebel zerstreut haben ${ }^{14}$. In all diesen Ausführungen Zwingers bemerkt man das Nachwirken der hippokratischen Schrift «Über Luft, Wasser und Ortslage», die der Verfasser gut kannte.

Zwinger kommt dann auf die Diät und mäßige Lebensweise zurück, die viel zur Verlängerung des Lebens beiträgt. Er zitiert den Schülervers: Pone gulae metas, et erit tibi longior aetas ${ }^{15}$. Er erwähnt Hippokrates, der das 83. Jahr erreichte und der auf die Frage, wie er ein so hohes Alter erreicht habe, geantwortet haben soll, er sei nie voll gesättigt von der Tafel aufgestanden. Zwinger nennt dann noch ein anderes Beispiel, den kaiserli- 
chen Leibarzt Guilelmus Arragosius aus Toulouse, Chemiker und Alchimist, der vor hundert Jahren bei seinem Urgroßvater Jacob Zwinger gelebt habe, und dessen Bücher und Manuskripte er noch besitze. Arragosius habe alle Nahrung, die er zu sich genommen habe, abwägen lassen. Er sei dann 1610 in seinem 97. Altersjahr in Basel an der Pest gestorben. Weiter erzählt Zwinger, er sei im Juli 1705 von Luzern nach Bern gereist, und da habe er in Legnau gehört, es sei vor wenigen Wochen ein Bauer bestattet worden, der sein 104. Lebensjahr überschritten habe ${ }^{16}$. In Signau habe er einen Bauern, namens Hans Elleberger, gesehen, der ein rüstiger Neunziger gewesen sei. Vor einem halben Jahr habe er zum dritten Mal geheiratet und mit einer jungen Frau ein gesundes Töchterchen gezeugt. An der Taufe habe er sich mit Freunden betrunken und sich dabei die Schulter verrenkt, sei dann aber wieder glücklich genesen ${ }^{17}$. Vor einem Monat habe man in Basel den Schneider Ambrosius Salathé beerdigt, der fast 93 Jahre alt geworden sei. Auch Zwingers Großmutter mütterlicherseits lebe noch und sei auch schon fast neunzig Jahre alt und soweit gesund, abgesehen davon, daß sie an Arthritis leide ${ }^{18}$. Zwinger bringt noch viele weitere Beispiele langlebiger Menschen, aber darauf kann hier nicht näher eingegangen werden.

Die Quintessenz von Zwingers Ratschlägen ist immer wieder die Mäßigkeit, und hier steht er genau in der Tradition von Lodovico Cornaro, von dem eingangs gesprochen wurde. Zwingers Schrift ist für seine Zeit ein recht fortschrittliches Erzeugnis, bemüht er sich doch, durch entsprechende Ratschläge die Kindersterblichkeit zu senken. Man darf diesen Basler Arzt wohl mit Recht als einen der Vorläufer der Aufklärungsmedizin des 18. Jahrhunderts bezeichnen, wenn er auch im Hinblick auf sein gesamtes Lebenswerk weitgehend noch dem Barock verhaftet ist.

\section{Anmerkungen}

${ }^{1}$ Gruman, Gerald J., A History of ideas about the prolongation of life. Arno Press New York 1977 (Reprint). Transactions of the American Philosophical Society. New Series, Vol.56, Part 9, 1966. The American Philosphical Society December, 1966.

${ }^{2}$ Zwinger, Theodor, De adquirenda vitae longaevitate, Basel 1703. Die Arbeit wurde von Zwinger verbessert und vermehrt 1706 neu herausgegeben. Vgl. dazu Buess, H., Portmann, M. L. und Molling, P., Theodor Zwinger III (1658-1724). Ein Basler Arzt und Kinderarzt der Barockzeit. Studien zur Geschichte der Wissenschaften in Basel Fasc. 14, Basel 1962, S. 120-122.

${ }^{3}$ Zwinger, Theodor, De adquiranda vitae longaevitate, Basel 1706, S. $55 \mathrm{f}$.

${ }^{4}$ Ebenda S. 57. 
${ }^{5}$ Ebenda S. 58.

${ }^{6}$ Ebenda S. 59.

${ }^{7}$ Ebenda S. 60.

${ }^{8}$ Ebenda S. 61.

${ }^{9}$ Ebenda S. 62.

${ }^{10}$ Ebenda S. 62-63.

11 Ebenda S. 63-64.

${ }^{12}$ Ebenda S. 64-65.

${ }^{13}$ Ebenda S. 66.

${ }^{14}$ Ebenda S. 66-67.

${ }^{15}$ Ebenda S. 67.

16 Ebenda S. 33.

17 Ebenda S. 33-34.

${ }^{18}$ Ebenda S. 34.

\section{Summary}

When Zwinger had accomplished his thesis on longevity in 1703 he was not content. He therefore improved and enlarged it. He mainly stressed sobriety thus following the example of Lodovico Cornaro (1467-1566), a Venetian author. As a pediatrician he strove for diminution of infant mortality. His thesis thus heralded the Age of Enlightenment though Zwinger was still deeply rooted in baroque medicine.

Dr. Marie-Louise Portmann

Medizinhistorische Bibliothek

Klingelbergstraße 23

CH-4031 Basel 